\title{
Normal renal sonogram identifies renal colic patients at low risk for urologic intervention: a prospective cohort study
}

\author{
Justin W. Yan, MD, MSc ${ }^{*}$; Shelley L. McLeod, MSc*; Marcia L. Edmonds, MD, MSc ${ }^{*}$; \\ Robert J. Sedran, MD, MSc ${ }^{*}$; Karl D. Theakston, MD, MSc ${ }^{*}$
}

\section{ABSTRACT}

Introduction: Determining which patients with ureterolithiasis are likely to require urologic intervention is a common challenge in the emergency department (ED). The objective was to determine if normal renal sonogram could identify low-risk renal colic patients, who were defined as not requiring urologic intervention within 90 days of their initial ED visit and can be managed conservatively.

Methods: This was a prospective cohort study involving adult patients presenting to the EDs of a tertiary care centre with suspected renal colic over a 20 -month period. Renal ultrasonography (US) was performed in the diagnostic imaging department by trained ultrasonographers, and the results were categorized into four mutually exclusive groups: normal, suggestive of ureterolithiasis, visualized ureteric stone, or findings unrelated to urolithiasis. Electronic medical records were reviewed to determine if patients received urologic intervention within 90 days of their ED visit.

Results: Of 610 patients enrolled, 341 (55.9\%) had US for suspected renal colic. Of those, $105(30.8 \%)$ were classified as normal; none of these patients underwent urologic intervention within 90 days of their ED visit. Ninety (26.4\%) US results were classified as suggestive, and nine (10\%) patients received urologic intervention. A total of 139 (40.8\%) US results were classified as visualized ureteric stone, and 34 $(24.5 \%)$ patients had urologic intervention. Seven $(2.1 \%)$ US results were classified as findings unrelated to urolithiasis, and none of these patients required urologic intervention. The rate of urologic intervention was significantly lower in those with normal US results $(p<0.001)$ than in those with abnormal findings.

Conclusion: A normal renal sonogram predicts a low likelihood for urologic intervention within 90 days for adult ED patients with suspected renal colic.

\section{RÉSUMÉ}

Introduction: II est souvent difficile de discerner, au service des urgences (SU), les patients qui sont susceptibles de subir une intervention rénale parmi ceux qui souffrent d'une urétérolithiase. L'étude visait à déterminer si l'obtention d'un échogramme rénal normal permettait de reconnaître les cas de colique néphrétique à faible risque, définis par l'absence de nécessité d'une intervention rénale dans les 90 jours suivant la première consultation au SU et par la possibilité d'une prise en charge conservatrice.

Méthode: II s'agit d'une étude de cohortes, prospective, menée au SU d'un centre hospitalier de soins tertiaires, sur une période de 20 mois, chez des adultes souffrant vraisemblablement d'une colique néphrétique. Une échographie rénale (ER) a été effectuée au service d'imagerie diagnostique par des échographistes qualifiés, et les résultats ont été divisés en quatre groupes mutuellement exclusifs: normaux, évocateurs d'une urétérolithiase, révélateurs d'un calcul urétéral (visualisé), et non liés à une lithiase urinaire. Il y eu examen des dossiers médicaux électroniques afin de vérifier si des patients avaient subi une intervention rénale au cours des 90 jours suivant la consultation au SU. Résultats: Sur 610 patients sélectionnés, 341 (55.9\%) ont subi une ER pour une colique néphrétique présumée. Parmi ceuxci, 105 (30.8\%) ont obtenu des résultats normaux, et aucun d'entre eux n'a subi une intervention rénale au cours des 90 jours suivant la consultation au SU; chez 90 (26.4\%) patients, I'ER a donné des résultats évocateurs d'une urétérolithiase, et $9(10 \%)$ d'entre eux ont subi une intervention rénale; chez $139(40.8 \%)$ patients, I'ER a permis de visualiser un calcul urétéral, et $34(24.5 \%)$ d'entre eux ont subi une intervention rénale; enfin, chez 7 (2.1\%) patients, les résultats obtenus à I'ER n'étaient pas liés à une lithiase urinaire, et aucun d'entre eux n'a subi une intervention rénale. Le taux d'intervention rénale était significativement plus bas chez les patients ayant

From the *Division of Emergency Medicine and TSchulich School of Medicine and Dentistry, The University of Western Ontario, London, ON.

Correspondence to: Dr. Justin Yan, The University of Western Ontario - Emergency Medicine, E1-100 Westminster Tower, 800 Commissioners Road East, London, ON N6A 5W9; jyan2009@meds.uwo.ca.

This article has been peer reviewed.

(c) Canadian Association of Emergency Physicians 
obtenu des résultats normaux à I'ER $(p<0.001)$ que chez ceux ayant obtenu des résultats anormaux.

Conclusion: L'obtention d'un échogramme rénal normal permet de prévoir le faible risque d'intervention rénale dans les 90 jours suivant la consultation au SU, chez les adultes souffrant d'une colique néphrétique présumée.

Keywords: renal colic, ultrasonography, urolithiasis
Renal colic is a common emergency department (ED) presentation that affects approximately 1.2 million people in the United States annually. ${ }^{1}$ Due to its high sensitivity and specificity, computed tomography (CT) is the gold standard imaging modality for the diagnosis of urolithiasis. ${ }^{2-5} \mathrm{CT}$ also has the ability to identify other acute intra-abdominal pathology (e.g., abdominal aortic aneurysm, bowel pathology, appendicitis) that may mimic renal colic. ${ }^{6-8}$ At present, the American College of Radiology recommends CT as the initial imaging modality for acute-onset flank pain, particularly when there is a strong suspicion of stone disease. ${ }^{9}$

Although ultrasonography (US) has lower sensitivity and specificity in diagnosing urolithiasis compared to CT, ${ }^{10-12}$ it is an alternative imaging modality that does not expose the patient to ionizing radiation. ${ }^{13} \mathrm{~A}$ previous retrospective study reported that less than $1 \%$ of ED patients with suspected renal colic with a normal renal sonogram received urologic intervention (e.g., extracorporeal shock wave lithotripsy [ESWL], ureteral stent insertion, stone extraction) within 90 days of the initial presentation. ${ }^{14}$ However, that study had some inherent limitations due to its retrospective nature, including potential patient selection and spectrum bias.

While acknowledging its superior diagnostic accuracy, there is increasing concern about cumulative radiation exposure from imaging with $\mathrm{CT},{ }^{10}$ particularly since patients with renal colic are often young and undergo multiple diagnostic imaging tests over their lifetime..$^{15}$ In 2007, the American College of Radiology stated that the increased use of CT may result in a higher incidence of radiation-related cancers and that physicians should consider radiation exposure when selecting imaging tests for patients. ${ }^{16}$ Berrington de Gonzalez and colleagues estimated that 14,000 future cancers per year may be attributed to abdominal and pelvic CT scans in the United States. ${ }^{17}$

The objective of this study was to prospectively determine if a normal renal sonogram could predict a low likelihood of urologic intervention within 90 days of the initial ED visit in patients with suspected renal colic.

\section{METHODS}

\section{Study setting and population}

This was a prospective cohort study over a 20 -month period (October 2010 to June 2012) at an academic tertiary care centre consisting of two EDs with a combined annual census of 140,000 visits. Our centre is the major regional referral centre for urologic intervention within southwestern Ontario, with a catchment area of over 3 million patients. The study protocol was approved by the Health Sciences Research Ethics Board at The University of Western Ontario in London, Ontario.

All adult patients ( $\geq 18$ years) who presented to the ED with suspected renal colic were eligible for enrolment. Triage nurses screened all patients with chief complaints of abdominal pain, flank pain, or hematuria to determine if they were eligible for study enrolment. Attending physicians were also able to screen patients if they were missed at triage. Eligibility was confirmed by the attending physician, who also obtained informed written consent. Study enrolment did not alter the ED management of patients. To provide context and describe how US is used at our institution, characteristics and outcomes were collected for all patients with suspected renal colic, irrespective of the diagnostic imaging tests performed.

US was available from 8:00 am to 4:00 pm daily. At our institution, there is a specific renal US protocol in the diagnostic imaging department that consists of views of the kidneys, ureters, and bladder, as well as evaluation of ureteric jets and postvoid residual. Based on the attending physician's differential diagnosis, imaging of other intra-abdominal or pelvic structures, such as the aorta, ovaries, or biliary tree, may have been included. Patients who had a sonogram ordered after 4:00 pm returned the following day to receive their imaging test. US scans were performed by trained ultrasonographers and interpreted by staff radiologists. 


\section{Measurements and outcomes}

The results of renal imaging (US and CT) were divided into four mutually exclusive categories that were defined a priori as normal, ureteric stone seen, suggestive of ureterolithiasis, and other disease unrelated to urolithiasis. The "suggestive of ureterolithiasis" category included results where a stone was not seen in the ureter but hydronephrosis, perinephric fluid or periureteral stranding, intrarenal stones, or an abnormal or absent ureteric jet was present.

The primary outcome was the occurrence of urologic intervention, defined as ESWL, ureteral stent insertion, or ureteric stone extraction, within 90 days of the initial ED presentation. Secondary outcomes included admission to hospital from the ED, unplanned return visits for the same chief complaint, outpatient urology follow-up, missed days of school or work, and duration of analgesia use.

Trained research personnel extracted demographic and clinical parameters from paper and electronic charts using a standardized data collection tool. Patients were contacted by telephone 48 to 72 hours and 10 to 14 days after their initial ED visit to collect information on secondary outcomes. If patients were unable to be contacted by telephone, questionnaires were mailed. A 90-day electronic chart review was also conducted by research assistants to determine if patients had repeat ED visits, outpatient urology visits, or urologic intervention.

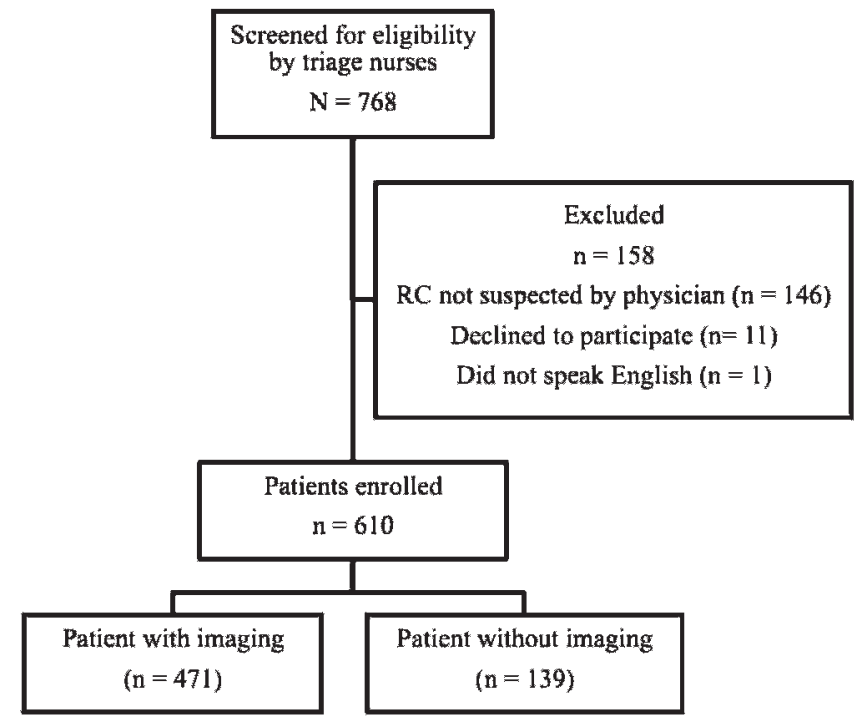

Figure 1. Flow diagram of included patients. $\mathrm{RC}=$ renal colic.

\section{Data analysis}

Data were entered directly into a study-specific Microsoft Excel database (Microsoft Corporation, Redmond, WA). Descriptive statistics were summarized using means and standard deviations or proportional differences with $95 \%$ confidence intervals where appropriate. Statistical analysis was conducted using Stata 13.0 (StataCorp LP, College Station, TX). Categorical outcomes were compared using a chisquare test, and continuous data were compared using an independent samples $t$-test.

\section{RESULTS}

Over the 20-month period, triage nurses screened 768 patients for eligibility. A total of 158 patients were excluded from the study, leaving 610 patients in the final analysis (Figure 1). Patient characteristics are summarized in Table 1.

There were 1,236 ED visits with a final diagnosis of urolithiasis over the study period. Of the 610 patients enrolled in the study, 471 (77.2\%) had some form of diagnostic imaging completed in the ED (Figure 2). Of those, $223(47.3 \%)$ had a ureteric stone visualized and $84(13.8 \%)$ patients underwent urologic intervention within 90 days of their initial ED visit.

A total of $341(55.9 \%)$ patients had US as the initial imaging modality for suspected renal colic. Of those, $263(77.1 \%)$ had US alone, 63 (18.5\%) had US and kidney-ureter-bladder (KUB) radiography, and 15 (4.4\%) had subsequent imaging with CT. Six patients had a stone seen on CT that was not visualized on US. The mean (SD) stone size was $3.5( \pm 1.3) \mathrm{mm}$. Among those 341 patients who had US, 105 (30.8\%) were classified as normal; none $(0 \%$; $95 \%$ CI $0.0-3.5)$ of these patients received urologic intervention within 90 days of the initial ED visit. Ninety (26.4\%) results were classified as suggestive, and nine (10\%; 95\% CI $5.3-$ 17.9) of these patients received urologic intervention. A total of 139 (40.8\%) results were classified as visualized ureteric stone, and $34(24.5 \%$; 95\% CI 18.1-32.2) of these patients had urologic intervention. Seven $(2.1 \%)$ results were classified as disease unrelated to urolithiasis; none $(0 \%$; $95 \%$ CI $0.0-3.5)$ of these patients received urologic intervention (Table 2). The final diagnoses for these patients are listed in Table 3. The rate of urologic intervention in those with a normal sonogram (0 of $105 ; 0 \%)$ was 


\begin{tabular}{lc|}
\hline \multicolumn{2}{l}{ Table 1. Patient demographics and characteristics } \\
\hline Characteristic & $N=610$ \\
\hline Male, $n(\%)$ & $370(60.7)$ \\
Mean (SD) age (yr) & $45.4(14.4)$ \\
Previous history, $n$ (\%) & \\
$\quad$ Renal colic & $264(43.3)$ \\
Urologic intervention for ureterolithiasis & $59(9.7)$ \\
Hypertension & $120(19.7)$ \\
Diabetes mellitus & $47(6.7)$ \\
Median (IQR) pain score at triage & $9(7-10)$ \\
Microscopic hematuria on urinalysis, $n$ (\%) & $479(78.5)$ \\
Median (IQR) creatinine ( $\mu$ mol/L) & $78(65-95)$ \\
CTAS, $n$ (\%) & \\
2 & $263(43.1)$ \\
3 & $300(49.2)$ \\
4 & $44(7.2)$ \\
5 & $3(0.5)$ \\
Admitted from ED, $n$ (\%) & $17(2.9)$ \\
\hline CTAS = Canadian Triage and Acuity Scale; ED = emergency department; IQR $=$ \\
interquartile range.
\end{tabular}

significantly lower $(p<0.001)$ than in those with abnormal results on a sonogram (43 of $236 ; 31.6 \%$ ).

A total of 105 patients had CT as the initial imaging modality. Of those, 70 patients had CT alone, with the remainder having additional imaging. Patients who had CT were more likely to be male, be older, have a history of hypertension and diabetes, and have a higher serum creatinine level than those who had US as the initial imaging modality. There was no difference with respect to previous history of renal colic, previous urologic intervention, acuity score at triage, vital signs, hematuria, pain score at triage, or proportion of patients admitted to hospital (Table 4).

Among the 105 patients who had CT, 15 (14.3\%) were classified as normal, and none $(0 \%$; $95 \%$ CI 0.0 $0.2 \%)$ received urologic intervention within 90 days of the initial ED visit. Ten (9.5\%) CT results were classified as suggestive, and none $(0 \%$; $95 \%$ CI 0.0 0.3 ) of these patients had urologic intervention. Seventy $(66.7 \%)$ results were classified as visualized ureteric stone, and 25 (35.7\%; 95\% CI 25.5-47.4) patients underwent urologic intervention. Ten (9.5\%) results were classified as disease unrelated to urolithiasis, and none (0\%; $95 \%$ CI $0.0-0.3)$ received urologic intervention (see Table 2). The final diagnoses for the 10 patients who were found to have disease unrelated to urolithiasis are presented in Table 3.

A total of $139(22.8 \%)$ patients did not have any imaging for suspected renal colic. Of those, 15 (10.8\%; 95\% CI 6.7-17.0) had a urologic intervention within 90 days of their initial ED visit, 12 of whom had a known previous history of urolithiasis and 2 others who had recent renal imaging prior to their ED visit.

Of the 610 patients enrolled in the study, 575 (94.3\%) were contacted at 48 to 72 hours and 10 to 14 days after their ED visit to determine analgesic use, missed days of school or work, and repeat visits to a health care provider. Fifteen $(2.6 \%)$ patients were admitted. A total of $150(26.1 \%)$ patients reported passing a stone within 72 hours, and 58 (10.1\%)

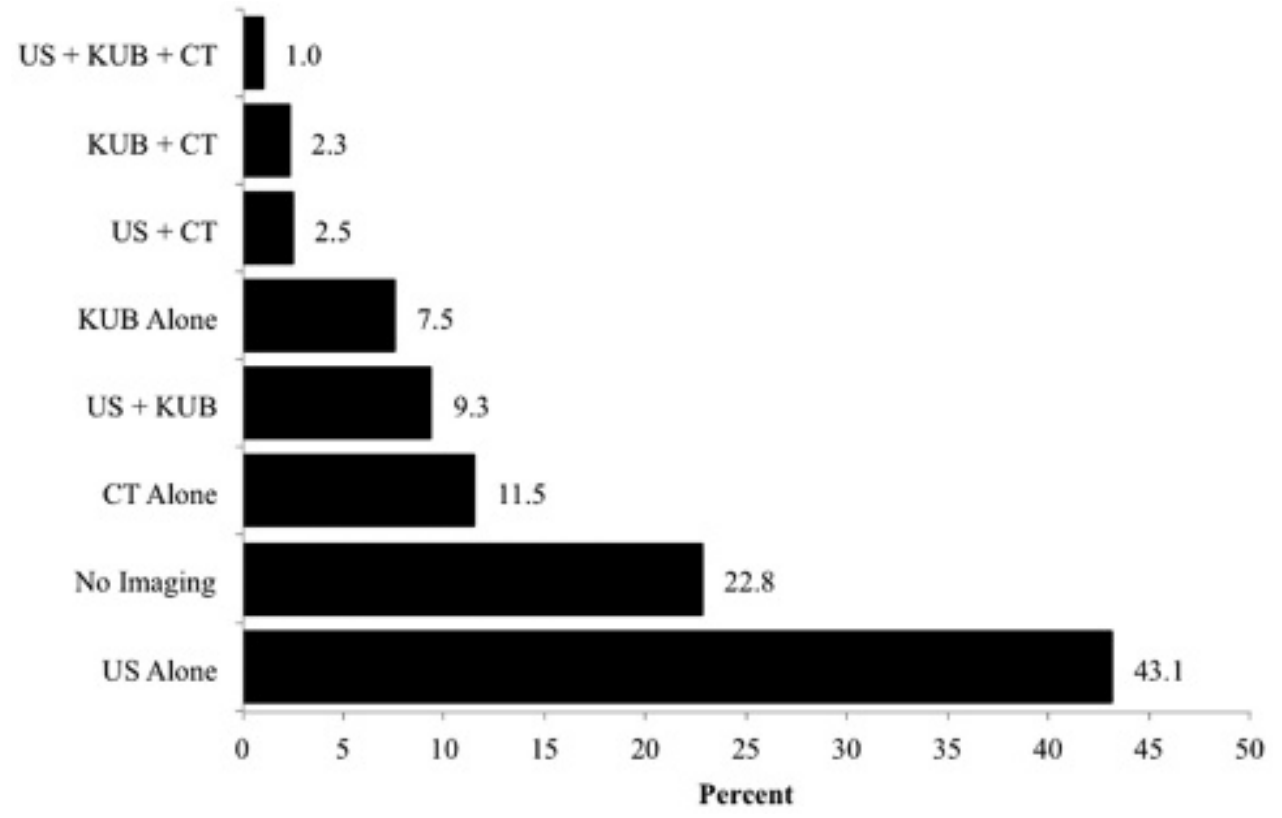

Figure 2. Diagnostic imaging tests performed in the emergency department for suspected renal colic. CT = computed tomography; KUB = kidney-ureter-bladder radiography; US = ultrasonography. 


\begin{tabular}{|c|c|c|c|c|}
\hline & US & $\begin{array}{l}\text { US urologic } \\
\text { intervention }\end{array}$ & $\mathrm{CT}$ & CT urologic intervention \\
\hline Examination findings & $n(\%)$ & $n(\%)$ & $n(\%)$ & $n(\%)$ \\
\hline Normal & $105(30.8)$ & $0(0)$ & $15(14.3)$ & $0(0)$ \\
\hline Ureteric stone & $139(40.8)$ & $34(24.5)$ & $70(66.7)$ & $25(36.8)$ \\
\hline Suggestive & $90(26.4)$ & $9(10.0)$ & $10(9.5)$ & $0(0)$ \\
\hline Other disease & $7(2.1)$ & $0(0)$ & $10(9.5)$ & $0(0)$ \\
\hline Total & $341(55.9)$ & $43(12.6)$ & $105(17.2)$ & $25(23.8)$ \\
\hline
\end{tabular}

additional patients reported passing a stone within 14 days. A total of 439 (76.3\%) patients required analgesia for a median (interquartile range [IQR]) duration of 4 (2-7) days, with 131 (29.8\%) still requiring analgesia at 10 to 14 days. A total of $275(47.8 \%)$ patients missed school or work for a mean (IQR) duration of 2 (1-4) days. A total of $270(47.0 \%)$ patients reported seeing another physician within 10 to 14 days (Table 5).

\section{DISCUSSION}

The results of this study confirm that a normal renal sonogram predicts a low risk of urologic intervention for ED patients with suspected renal colic within 90 days of their initial presentation. Kobayashi and colleagues reported that 6 of $238(2.5 \%)$ patients who had negative or equivocal results on the initial $x$-ray or

Table 3. Final diagnosis for 17 patients who underwent US or CT imaging with diseases unrelated to urolithiasis

\begin{tabular}{ll}
\hline Diagnosis & Number of patients \\
Imaging: US & 1 \\
Neoplastic disease & 1 \\
Medullary sponge kidney & 1 \\
Pelvic cysts & 1 \\
Cholelithiasis and UTI & 1 \\
Ureterocele and pyelonephritis & 1 \\
Appendicitis & 1 \\
Pregnancy & \\
Imaging: CT & 3 \\
Neoplastic disease & 3 \\
Diverticular disease & 2 \\
Pyelonephritis & 1 \\
Ovarian cyst & 1 \\
Parapelvic cysts and diverticulosis & CT \\
CT = computed tomography; US = ultrasonography; UTI = urinary tract infection.
\end{tabular}

sonogram received urologic intervention within 30 days. ${ }^{18}$ Recently, Edmonds and colleagues reported findings from a retrospective medical record review for all adult patients who had ED-ordered renal US for suspected renal colic over a 1 -year period. ${ }^{14}$ The authors concluded that a normal renal sonogram predicted a low likelihood $(<1 \%)$ of urologic intervention within 90 days. However, patients who underwent primary CT or those who did not have any imaging were not captured. It is possible that physicians have preferentially selected CT for patients thought to be at higher risk for complicated stone disease or other significant pathology. Physicians may have chosen to forego all imaging for patients with suspected renal colic if they had a typical presentation with no concerning features, such as fever or solitary kidney, as suggested by Lindqvist and colleagues. ${ }^{19}$ To address these concerns, the present study attempted to enrol patients with any presenting complaint that could be related to urolithiasis.

Urolithiasis is generally a benign disease with few complications. ${ }^{11}$ Despite being a non-life-threatening illness, the burden of disease of renal colic is significant. The adverse outcomes affect patients and the health care system beyond what may be seen with an isolated ED visit. Patients with renal colic are likely to undergo multiple imaging investigations over their lifetime, including CT. ${ }^{15}$ There is increasing concern about cumulative radiation exposure from CT imaging, particularly since previous studies have suggested that this may increase the long-term risk of developing cancer. ${ }^{10,16}$

A recent study reported that emergency physicians consider the likelihood of other significant intraabdominal pathology when selecting the primary imaging modality for suspected renal colic. ${ }^{20}$ Although 
Table 4. Characteristics of patients receiving US alone versus CT alone as first-line imaging modality for suspected urolithiasis

\begin{tabular}{|c|c|c|c|}
\hline & US alone & CT alone & \\
\hline Characteristic & $n=263$ & $n=70$ & $\Delta 95 \% \mathrm{Cl}$ \\
\hline Male & $141(53.6 \%)$ & $51(72.9 \%)$ & $19.3(6.4,30.1)$ \\
\hline Mean (SD) age (yr) & $43.7(14.2)$ & $61.5(12.4)$ & $17.8(14.2,21.5)$ \\
\hline \multicolumn{4}{|l|}{ Previous history } \\
\hline Renal colic & $91(34.6 \%)$ & $21(30.0 \%)$ & $4.6(-8.2,15.8)$ \\
\hline Urologic intervention for ureterolithiasis & $16(6.1 \%)$ & $3(4.3 \%)$ & $1.8(-6.1,6.4)$ \\
\hline Hypertension & $40(15.2 \%)$ & $26(37.1 \%)$ & $21.9(10.5,34.3)$ \\
\hline Diabetes mellitus & $17(6.5 \%)$ & $10(14.3 \%)$ & $7.8(0.5,18.1)$ \\
\hline Febrile at triage (temperature $\geq 38.0^{\circ} \mathrm{C}\left[100.4^{\circ} \mathrm{F}\right]$ ) & 0 & 0 & \\
\hline Tachycardic (heart rate $>100$ bpm) & $28(10.6 \%)$ & $7(10.0 \%)$ & $0.6(-9.1,7.3)$ \\
\hline Median (IOR) pain score at triage & $8(7-10)$ & $8(8-10)$ & $0(-0.7,0.7)$ \\
\hline Microscopic hematuria on urinalysis & $212(80.6 \%)$ & $52(74.3 \%)$ & $6.3(-3.9,18.4)$ \\
\hline Median (IOR) creatinine ( $\mu \mathrm{mol} / \mathrm{L})$ & $75(63-92)$ & $89(72-105)$ & $14(5.2,22.8)$ \\
\hline \multicolumn{4}{|l|}{ CTAS } \\
\hline 2 & $104(39.5 \%)$ & $26(37.1 \%)$ & $2.4(-10.6,14.4)$ \\
\hline 3 & $132(50.2 \%)$ & $42(60.0 \%)$ & $9.8(-3.3,22.0)$ \\
\hline 4 & $26(9.9 \%)$ & $2(2.9 \%)$ & $7.0(-0.5,11.7)$ \\
\hline 5 & $1(0.4 \%)$ & 0 & $0.4(-4.8,2.1)$ \\
\hline
\end{tabular}

CT is the preferred imaging modality for diagnosing some common pathologies (e.g., diverticular disease, bowel obstruction), US is the preferred choice for others (e.g., ovarian or other gynecologic pathology, biliary tract disease). ${ }^{21}$ The results of this study suggest that US can be used as an initial screening tool for most patients with suspected urolithiasis. Initial imaging with CT may be warranted if there is sufficient clinical suspicion of certain other disease processes.

Some believe that significant pathology may be missed when US is selected as the initial diagnostic

\begin{tabular}{|c|c|}
\hline Physician type & $n(\%)$ \\
\hline Family physician or walk-in clinic & $141(52.2)$ \\
\hline Urologist & $77(28.5)$ \\
\hline Another emergency physician & $22(8.1)$ \\
\hline Family physician or walk-in clinic and urologist & $5(1.9)$ \\
\hline $\begin{array}{l}\text { Family physician or walk-in clinic and another } \\
\text { emergency physician }\end{array}$ & $7(2.6)$ \\
\hline Urologist and another emergency physician & $8(3.0)$ \\
\hline $\begin{array}{l}\text { Family physician or walk-in clinic and another } \\
\text { emergency physician and urologist }\end{array}$ & $1(0.4)$ \\
\hline Other physician & $9(3.3)$ \\
\hline
\end{tabular}

imaging modality. In this study, two patients had a normal sonogram (no hydronephrosis, perinephric fluid, or bilateral urinary jets seen) and a stone identified on a CT scan. Both stones were small $(<5 \mathrm{~mm})$ and did not require urologic intervention within 90 days. One patient had a rectal mass visualized on US that was subsequently confirmed by CT. There were no significant intra-abdominal pathologies missed on US that were detected during the 90-day follow-up period.

When a stone cannot be visualized with US, other findings suggestive of obstruction, such as hydronephrosis, abnormal ureteric jets, or perinephric fluid, can aid in establishing the diagnosis. ${ }^{14,22}$ In this study, $10 \%$ of patients who had US findings suggestive of ureterolithiasis received urologic intervention within 90 days. This is similar to the intervention rate $(6.8 \%)$ reported by Edmonds and colleagues for patients with suggestive findings on renal US. ${ }^{14}$ However, the urologic intervention rate for patients where a stone was visualized on US was much higher in the present study $(24.5 \%$ v. $6.2 \%)$. This suggests a change in institutional management of ureteric stones given that patient demographics, stone size, and location were similar in both studies and both were done at the same centre. 
Uncertainty remains with respect to the appropriate management of patients who have US findings suggestive of obstruction but have no stone visualized. In the present study, 12 of $90(13.3 \%)$ patients with a suggestive sonogram went on to have CT, and 2 $(2.2 \%)$ ultimately had urologic intervention within 90 days of the initial presentation. Future studies should focus on the appropriate clinical pathway for these patients, specifically if they should have expedited CT or prompt urologic follow-up.

\section{LIMITATIONS}

This study was conducted at a single centre consisting of two tertiary care EDs, and the results may not be generalizable to other settings where US for the diagnosis of ureterolithiasis is not routinely used. Some of the patients enrolled may have sought urologic consultation outside our centre's catchment area. However, our institution is the major regional referral centre within southwestern Ontario for urologic intervention, resulting in a relatively closed medical system.

Because US is operator dependent, its diagnostic accuracy varies between institutions. In this study, US was performed by trained ultrasonographers and interpreted by staff radiologists. Therefore, caution should be exercised regarding extrapolation of the results of this study to bedside (point-of-care) ED US. Furthermore, the threshold for urologic consultation from the ED and surgical intervention rates for renal colic may vary from centre to centre depending on the region or country. The decision of whether and when to perform intervention for ureterolithiasis may depend in part on the urologist, and the specific clinical indication for intervening was not captured as part of this study.

In the present study, the final diagnosis of ureterolithiasis was based on a clinical decision made by the emergency physician and not necessarily confirmed by imaging. It is possible that some patients with this final diagnosis did not have stones at all, contributing to some extent to the low rate of intervention. When imaging results were normal, patients may have had undetected small stones (i.e., not visualized) and passed without intervention or complication.

Selection bias may have been a factor in this study. In our study, more complex patients (older, diabetic, abnormal renal function) were more likely to be imaged with CT. Eligibility for enrolment was based on a clinical suspicion of renal colic at triage. Although triage nurses and physicians were asked to consider all-comers with any presenting complaint that could be related to urolithiasis, the majority of patients enrolled likely had a classic presentation. Patients with atypical signs and symptoms may not have been enrolled. Caution should be used if the results from this study are extrapolated to a broader population of patients with more atypical symptoms. However, given that this was a prospective, pragmatic study, we do not believe that the external validity of our conclusion is compromised.

\section{CONCLUSIONS}

Although US has less diagnostic accuracy compared to CT, patients with a clinical diagnosis of renal colic and a normal renal sonogram are unlikely to require urologic intervention within 90 days of their initial ED visit and can confidently be managed conservatively with appropriate analgesia and clinical follow-up. In combination with clinical assessment, a renal US protocol can accurately identify this low-risk group, decreasing the number of CT scans and associated ionizing radiation in patients with suspected renal colic.

Competing interests: None declared.

\section{REFERENCES}

1. Wolf JS Jr, Schwartz BF. Nephrolithiasis. Available at: http:// emedicine.medscape.com/article/437096-overview (accessed July 23, 2012).

2. Kenney PJ. CT evaluation of urinary lithiasis. Radiol Clin North Am 2003;41:979-99, doi:10.1016/S0033-8389(03) 00067-8.

3. Tamm EP, Silverman PM, Shuman WP. Evaluation of the patient with flank pain and possible ureteral calculus. Radiology 2003;228:319-29, doi:10.1148/radiol.2282011726.

4. Gottlieb RH, La TC, Etkurk EN, et al. CT in detecting urinary tract calculi: influence on patient imaging and clinical outcomes. Radiology 2002;225:441-9, doi:10.1148/ radiol.2252020101.

5. Kirpalani A, Khalili K, Lee S, et al. Renal colic: comparison of use and outcomes of unenhanced helical CT for emergency investigation in 1998 and 2002. Radiology 2005; 236:554-8, doi:10.1148/radiol.2362040887.

6. Roberts JR. Kidney stone or aortic catastrophe? Emerg Med News 2006;28:31-4, doi:10.1097/00132981-200604000-00025.

7. Catalano O, Nunziata A, Sandomenico F, et al. Acute flank pain: comparison of unenhanced helical CT and ultrasonography in detecting causes other than ureterolithiasis. Emerg Radiol 2002;9:146-54. 
8. Miller OF, Kane CJ. Unenhanced helical computed tomography in the evaluation of acute flank pain. Curr Opin Urol 2000;10:123-9, doi:10.1097/00042307-200003000-00013.

9. American College of Radiology. Appropriateness criteria. Available at: http://www.acr.org/ /media/ACR/Documents/ AppCriteria/Diagnostic/AcuteOnsetFlankPainSuspicionStone Disease.pdf (accessed May 24, 2013).

10. Brenner DJ, Hall EJ. Computed tomography - an increasing source of radiation exposure. N Engl f Med 2007;257:227784, doi:10.1056/NEJMra072149.

11. Teichman JM. Acute renal colic from ureteral calculus. $N$ Engl 7 Med 2004;350:684-93, doi:10.1056/NEJMcp030813.

12. Ripolles T, Agramunt M, Errando J, et al. Suspected ureteral colic: plain film and sonography vs unenhanced helical CT. A prospective study in 66 patients. Eur Radiol 2004;14:12936, doi:10.1007/s00330-003-1924-6.

13. Patlas M, Farkas A, Fisher D, et al. Ultrasound vs CT for the detection of ureteric stones in patients with renal colic. $\operatorname{Br} 7$ Radiol 2001;74:901-4.

14. Edmonds ML, Yan JW, Sedran RS, et al. The utility of renal ultrasonography in the diagnosis of renal colic in emergency department patients. CFEM 2010;12:201-6.

15. Broder J, Bowen J, Lohr J, et al. Cumulative CT exposures in emergency department patients evaluated for suspected renal colic. 7 Emerg Med 2007;33:161-8, doi:10.1016/ j.jemermed.2006.12.035.
16. Amis ES Jr, Butler PF, Applegate KE, et al. American College of Radiology white paper on radiation dose in medicine. $7 \mathrm{Am}$ Coll Radiol 2007;4:272-84, doi:10.1016/j.jacr.2007.03.002.

17. Berrington de Gonzalez A, Mahesh M, Kim KP, et al. Projected cancer risks from computed tomographic scans performed in the United States in 2007. Arch Intern Med 2009;169:2071-7, doi:10.1001/archinternmed.2009.440.

18. Kobayashi T, Nishizawa K, Watanabe J, et al. Clinical characteristics of ureteral calculi detected by nonenhanced computerized tomography after unclear results of plain radiography and ultrasonography. 7 Urol 2003;170:799-802, doi:10.1097/01.ju.0000081424.44254.45.

19. Lindqvist K, Hellstrom M, Holmberg G, et al. Immediate versus deferred radiological investigation after acute renal colic: a prospective randomized study. Scand 7 Urol Nephrol 2006;40:119-24, doi:10.1080/00365590600688203.

20. Yan JW, McLeod SL, Edmonds ML, et al. Factors affecting choice of imaging investigations for patients with suspected renal colic in the emergency department. CFEM 2012;14 Suppl 1:S31.

21. Sternbach G. Abdominal ultrasound. Ann Emerg Med 1986; 15:295-9, doi:10.1016/S0196-0644(86)80568-6.

22. Watkins S, Bowra J, Sharma P, et al. Validation of emergency physician ultrasound in diagnosing hydronephrosis in ureteric colic. Emerg Med Australas 2007;19:18895, doi:10.1111/j.1742-6723.2007.00925.x. 